\title{
LA INTEGRACIÓN DEL CAMBIO CLIMÁTICO EN LA PLANIFICACIÓN DE LOS RIESGOS AMBIENTALES EN EL LITORAL CATALÁN
}

\author{
Elisabet ROCA BOSCH${ }^{1}$, Míriam VILLARES JUNYENT ${ }^{1}$ \\ ${ }^{1}$ Institut de Sostenibilitat. Universitat Politècnica de Catalunya. \\ Elisabet.roca@upc.edu, miriam.villares@upc.edu
}

\section{RESUMEN}

Los riesgos que afectan el litoral como la erosión de playas, la inundación y la contaminación marina se pueden ver agravados por los efectos del cambio climático. Tener mecanismos de respuesta adecuados requiere un sistema de planeamiento integrado, multinivel, holístico, multi-riesgo. El objetivo de esta investigación, dentro del Proyecto Pairis-Clima, es analizar el sistema de planeamiento para hacer frente a los riesgos que afectan al litoral catalán y evaluar la integración del cambio climático.

Los resultados apuntan a una planificación de la prevención y de la emergencia de los riesgos fragmentada y poco coordinada en diferentes niveles institucionales y sectoriales. La componente del cambio climático le añade complejidad a la cuestión. Todavía ausente en la planificación de la emergencia, se encuentra de manera explícita pero sólo a nivel formal en la normativa costera, mientras que en la planificación hidrológica y en la evaluación ambiental estratégica los avances parecen más significativos. Actualmente dos leyes catalanas en redacción, la del cambio climático y de litoral abren nuevas expectativas para mejorar en este campo.

Palabras clave: riesgo costero, integración normativa, litoral catalán, planeamiento normativo.

\begin{abstract}
Those risks affecting our coasts such as beach erosion, flooding and marine pollution can be aggravated by climate change. Having adequate response mechanisms requires an integrated, holistic, multi-risk planning system. It should incorporate different phases of risk management, from prevention to emergency, with the participation of a wide range of authorities. This research aims to analyze the statutory planning system to cope with risks affecting the Catalan coast (northwestern of the Mediterranean Basin) and evaluate the level of integration of climate change in this legislation.

The results show a fragmentation and a poor coordination between the prevention planning and the emergency planning and the different institutional and sectoral levels. The component of climate change adds complexity to the issue at stake. Still absent in emergency planning, it is explicitly but only on a formal level in the coastal legislation, while in the water planning and strategic environmental assessment advances seem more significant. Currently two Catalan laws in elaboration, the climate change law and coastal planning law generate new expectations to improve in this field.
\end{abstract}

Key words: coastal risk, legal integration, Catalan coast, statutory planning 


\section{INTRODUCCIÓN Y OBJETIVOS}

Los riesgos que afectan el litoral como la erosión de playas, la inundación y la contaminación marina se pueden ver agravados por los efectos del cambio climático. Tener mecanismos de respuesta adecuados requiere un sistema de planificación integrado, multinivel, holístico, multi-riesgo que incorpore las diferentes fases de gestión del riesgo con la participación de actores de naturaleza diversa (Ribot, 2008). El vector del cambio climático añade más complejidad a la gobernanza del riesgo costero lo que requiere dotar al sistema planificador de más capacidad de adaptación para hacer frente al futuro (Dietz et al. 2003; Folke et al. 2005). Dotar de una visión más adaptativa la planificación del riesgo, implica trabajar con procesos que enfatizan el aprendizaje continuo, reconocimiento explícito de la complejidad y las incertidumbres (Allen et al. 2011) inherentes al sistema costero .Por lo tanto, los instrumentos de planificación deben ser lo suficientemente flexibles para ir incorporando el conocimiento que se genera en la ciencia y en la práctica. Al final se añade, un elemento clave del enfoque adaptativo: la participación social (Norton, 2005).

El objetivo de esta comunicación es analizar el sistema de planeamiento actual para hacer frente a los riesgos que afectan al litoral Catalán y evaluar cómo se integra la normativa del vector del cambio climático. La comunicación se enmarca en el Proyecto El Paisaje del Riesgo Costero en el litoral catalán. La influencia del cambio climático (Pairis-Clima) cuyo objetivo es analizar el paisaje del riesgo costero a lo largo del litoral catalán bajo diferentes escenarios climáticos a escala regional y, proponer medidas de gestión del riesgo a largo plazo que tengan en cuenta el efecto del cambio climático. El objetivo es identificar como el efecto del cambio climático se propaga en el sistema a través de su influencia en las diferentes componentes. Su uso permitirá al gestor valorar la importancia relativa del cambio climático en los diferentes procesos y consecuencias, valorando la necesidad de inclusión en la toma de decisiones en los procesos de gestión del riesgo.

La metodología que se ha seguido parte de un inventario de los instrumentos de planificación en materia de gestión de riesgos costeros, ordenación del territorio y protección del litoral. Este trabajo de revisión documental se ha complementado con una docena de entrevistas a responsables político-técnicos de la administración (estatal, autonómica y local) ha permitido aportar la opinión experta del planificador y del gestor para obtener una valoración del status quo de la gestión de los riesgos costeros y de cómo se está integrando el vector del cambio climático en este sistema normativo. En definitiva, el objetivo es identificar debilidades y oportunidades de mejorar teniendo como referencia los principios de la gestión adaptativa.

La comunicación parte del análisis de la planificación de los riesgos ambientales en el litoral catalán como punto de partida para valorar como se está produciendo la integración del vector del cambio climático en esta planificación.

\section{LA PLANIFICACIÓN EN MATERIA DE RIESGOS LITORALES}

Dentro del ciclo de gestión de los riesgos naturales podemos señalar tres etapas, la preparación, la respuesta y la recuperación, en relación al momento que tiene lugar el fenómeno natural. La planificación se produce de manera anticipada al evento, en la etapa de la preparación que puede clasificarse según dos momentos: la prevención y la emergencia. La planificación de la prevención es la que integra los riesgos naturales en la ordenación del territorio y el urbanismo (como por ejemplo la delimitación de zonas de inundables y su consideración en los planes territoriales o los planes de ordenación urbana municipal) y la planificación de la emergencia de naturaleza reactiva, la que acciona los protocolos 
y mecanismos para hacer frente a situaciones de emergencia y paliar los efectos que se puedan producir (por ejemplo en los planes del Inuncat, Procicat y Camcat).

La procedencia diversa de los riesgos que afectan al litoral Catalán, se reflejan en un marco competencial complejo constituido por una superposición de normativa y planeamiento sectorial y procedente de administraciones de diferentes niveles competenciales. Para poder proceder a la identificación y análisis de los instrumentos de planificación se han inventariado y clasificado en función del nivel administrativo y de la etapa en la que se despliegan planes y normativa.

A nivel estatal, los principales instrumentos de planificación de recién o en fase de elaboración son: la Ley de Protección y Uso Sostenible del Litoral y de Modificación de la Ley de Costas (2013), la Estrategia Marina para la Demarcación Levantino-Balear, el Plan Estatal de Protección del Medio Marino contra la Contaminación (Plan Ribera) y la Estrategia para la Adaptación de la Costa a los Efectos del Cambio Climático. Todos ellos a manos del Ministerio de Agricultura Alimentación y Medio Ambiente (MAGRAMA). Tanto la Ley de Costas como el Plan Ribera, integran la prevención como la emergencia de los respectivos riesgos que gestiona: la primera, la protección del Dominio Público Marítimo-Terrestre (DPMT) a los efectos de temporales, subida del nivel del mar y procesos erosivos y, el segundo, la calidad ambiental y el riesgo de contaminación marina.

A nivel autonómico, la titularidad de los instrumentos que gestionan la prevención y los que gestionan la emergencia está dividida. La planificación de la emergencia está en manos de Protecció Civil de la Generalitat de Catalunya responsable de Pla de Protecció Civil de Catalunya (PROCICAT), el Plan de Contaminación Marina de Catalunya (CAMCAT), el Plan de Inundaciones de Catalunya (INUNCAT), mientras que la ordenación del territorio, a través de los Plans Territorials está en manos del Departament de Territori i Sostenibilitat. Finalmente, la escala local desarrolla una función preventiva a través de la planificación urbanística y tiene un papel muy relevante en la gestión de la emergencia.

\begin{tabular}{|c|c|c|}
\hline & Prevención & Emergencia \\
\hline Estatal & \multicolumn{2}{|c|}{$\begin{array}{l}\text { Estrategia Marina } \quad \text { Ley de Costas } \\
\text { Estrategia para la adaptación } \\
\text { de la costa a los efectos del cambio climático } \\
\text { Plan Hidrológico del Ebro } \\
\text { Pla d'Usos dels Ports d'Interés general }\end{array}$} \\
\hline Catalan & $\begin{array}{l}\text { Plans Territorials } \\
\text { Pla de Ports Pla de Gestió } \\
\text { Estrategia Catalana de Canvi }\end{array}$ & $\begin{array}{l}\text { nques Internes de Cat } \\
\text { PROCICAT } \\
\text { CAMCAT } \\
\text { INUNCAT }\end{array}$ \\
\hline Municipal & $\begin{array}{l}\text { POUM - Plan de Ordenación } \\
\text { Urbana Municipal }\end{array}$ & $\begin{array}{l}\text { Pla bàsic d'emergència municipal } \\
\text { (PAEM) } \\
\text { Plans d'actuació (PAM) municipal } \\
\text { Plans específic municipal (PEM) }\end{array}$ \\
\hline PARTICULAR & & Plans d'Autoprotecció (PAU) \\
\hline
\end{tabular}

Tabla 1: Listado de planes vinculados a la gestión de riesgos litorales 
Este listado de planeamiento como se observa en la tabla 1, está fragmentado entre diferentes niveles institucionales y departamentos dificultando la coordinación especialmente en momentos en que la distancia política es mayor. Si bien es cierto, que la planificación hidrológica y la de puertos reúnen en un mismo instrumento, ya sean los planes de cuenca o los planes de usos de los puertos, una visión multi-riesgo incorporando, erosión, contaminación, inundación, ya sea en sus fases de prevención como de gestión. Sin embargo, la unidad territorial de análisis es o bien la cuenca o bien la actividad portuaria y por lo tanto, no resulta suficiente para aproximarse a la realidad litoral. Además, si atendemos al tipo de riesgo la tradición planificadora es bastante diferente. Mientras en el riesgo de inundación, la participación institucional es amplia y multiescalar, en el riesgo de erosión, una componente muy relevante del riesgo global costero, la competencia se centraliza en el estado dejando poco margen de actuación a niveles administrativos inferiores.

Por ello se convierte en indispensable establecer mecanismos de coordinación y relación entre estos instrumentos si se quiere dotar al litoral de un enfoque más estratégico e integrado. Sólo hemos encontrado en el planeamiento hidrológico está vocación más holística y una visión más integrada de los riesgos, aunque como veremos más adelante, existe una iniciativa del gobierno catalán muy incipiente para elaborar un plan de ordenación del litoral que podría convertirse en el instrumento estratégico, de cohesión y coordinación que requerimos.

Y a toda esta complejidad hay que incorporarle las previsiones de cambio climático y, por lo tanto, estudiar cómo se están planificando las estrategias de adaptación.

\section{LA INTEGRACIÓN DEL CAMBIO CLIMÁTICO EN LA NORMATIVA ACTUAL Y PERSPECTIVAS DE FUTURO}

Para alcanzar los objetivos de este trabajo se ha sometido este sistema de planeamiento a la valoración de responsables institucionales que nos ha permitido hacer una valoración cualitativa del nivel de integración del vector del cambio climático. También se han identificado oportunidades para un mayor reconocimiento normativo del cambio climático en un futuro próximo.

Las grandes directrices sobre cómo debe producirse esta integración están en manos de las diferentes oficinas de cambio climático. A nivel estatal, desde la Oficina Española de Cambio Climático y el MAGRAMA se está elaborando la Estrategia de Adaptación de la Costa al Cambio Climática (EACCC), iniciativa interesante que actualmente se encuentra en fase de evaluación ambiental. El documento actualmente es de naturaleza estratégica aportando escenarios regionales pero de difícil concreción para aplicar un proyecto específico.

Desde la Ley de Costas, principal instrumento legal y de ordenación, en su última modificación, la del 2013, se incorpora la exigencia de considerar el cambio climático en la redacción de nuevos proyectos. Sin embargo, a pesar del desarrollo de la EACCC, resulta poco operativa para el nivel de proyecto y es necesario especificar los escenarios por tramos de litoral y hacer evaluaciones más locales para que la Ley y la EACCC resulten efectivas. Más allá de esta intención aparente y con poca fuerza legal, la normativa prioriza un enfoque basado en la rigidificación y la ampliación de los regímenes concesionarios, hecho que evidencia cierta incoherencia con las estra- 
tegias de adaptación litoral que requeriría una apuesta más fuerte por la restauración y la conservación dunar y de ecosistemas litorales. Por lo tanto, el proceso es lento y se identifica poco con la visión adaptativa, a parte debería buscar más complicidades en todos los agentes territoriales implicados a los efectos del cambio climático como la subida del nivel del mar y efectos de temporales.

A nivel catalán desde la Oficina Catalana de Canvi Climàtic (OCCC) se elaboró la Estratègia Catalana d'Adaptació al Canvi Climàtic (ESCACC) Horitzó 2013-2020 que, sin abordar el tema de la costa de manera directa y explícita, pone sobre papel la necesidad de concretar planes, instrumentos que establezcan medidas de actuación urgentes y prioritarias, relativas a sectores como el turismo, la agricultura, la pesca... . Sin embargo, hay falta de concreción también porque no se especifican qué planes deben redactarse y en qué horizonte temporal. La ESCACC también reconoce que la integración de la adaptación del cambio climático en la legislación sectorial es un proceso complejo que requiere de mucha consciencia política y de nuevas metodologías.

Por lo tanto, la integración del cambio climático en la normativa y planificación vigente es un gran reto que todavía se encuentra en una etapa muy incipiente. Se ha ido produciendo estos últimos años a través de numerosas normativas sectoriales como la Evaluación Ambiental Estratégica (EAE). Por ejemplo, en la ordenación territorial y la urbanística, se exige a través de la EAE la previsión de riesgos derivados del cambio climático. En los Plans Territorials Parcials (la ordenación territorial por grandes áreas funcionales), estos riesgos se integran en la clasificación de suelo no urbanizable de protección especial. También desde estos se recomiendan medidas de adaptación diversas como la creación de cordones dunares y la protección de ecosistemas frágiles. Sin embargo, la competencia en muchos casos de estas medidas supera el ámbito del plan y recae en niveles administrativos superiores no sujetos a las determinaciones del plan.

En la planificación hidrológica, la más compleja y transversal en estos momentos, se referencia de manera explícita especialmente en los Planes de Gestión de Riesgos de Inundación el vector del cambio climático. Sin embargo, más allá de reconocer su existencia y alertar de sus riesgos, a la hora de cuantificar los fenómenos, la fiabilidad es baja, sobre todo cuando se quieren regionalizar los impactos, y por lo tanto, tiene pocos efectos operativos.

Finalmente, en la planificación de la emergencia se responde a unos objetivos que no necesariamente incorporan la variable climática. Por ello, no hay rastro del cambio climático en los instrumentos que se derivan de Protecció Civil, es decir, en los planes que tradicionalmente han servido para gestionar el momento de la emergencia ya sea a nivel autonómico como a nivel local.

Esta situación todavía muy precaria en cuanto a la consideración legal del cambio climático se pretende abordar a nivel catalán a través del impulso de dos nuevas normativas que pueden representar una gran oportunidad para cubrir los vacíos identificados anteriormente. Se tratan de la Llei d'Ordenació del Litoral y la Llei de Canvi Climàtic.

La primera se encuentra en una fase muy primigenia, en redacción y con mucho recorrido todavía por hacer. Esto le concede una oportunidad para solventar las vaguedades de la Ley de Costas en cuanto al cambio climático y se favorezca la creación 
de un instrumento estratégico tan necesario como el Plan de Ordenación Litoral y su desarrollo reglamentario. Confiamos que sirva para integrar los escenarios de cambio climático tanto la ordenación de los usos actuales como en la redacción de nuevos proyectos en el litoral catalán, aunque se prevé el escollo competencial del DPMT.

Un poco más avanzada se encuentra, la Llei de Canvi climàtic que se está elaborando desde la OCCC, ahora en fase de anteproyecto. Su objetivo, tal y como se contempla en su artículo 7 es obligar a las administraciones competentes a introducir el vector del cambio climático en la planificación y programación sectorial. Hasta el momento la OCCC sólo actúa ejerciendo presión sobre las diferentes administraciones sectoriales para integrar el cambio climático pero con la ley se quiere tener la fuerza normativa necesaria para que gane significación este tema y se traduzca en medidas operativas. De nuevo, el principal problema sin embargo reside en la distancia institucional, ya que la gestión del DPMT está en manos del MAGRAMA y la OCCC tiene un alcance autonómico. Esto ya se visualiza en la Comisión de Coordinación de Políticas de Cambio Climático, órgano consultivo y de coordinación entre el ministerio y representantes territoriales tiene una vocación meramente informativa. Con una reunión anual apenas cumple con la función que por decreto se le ha sido asignada.

\section{CONCLUSIONES}

En nuestro sistema jurídico contamos con numerosos instrumentos de carácter sectorial que regulan de manera dispersa y fragmentaria los aspectos vinculados a los riesgos costeros. Su complejidad se hace patente en la distribución competencial, tanto sectorial como multinivel de los sistema de planeamiento y las instituciones responsables. En parte responde al origen diverso de los riesgos que afectan al litoral (mar, tierra, aire), su escala espacial (de locales a transfronterizo) y temporal (inmediatos vs. Procesos lentos). Esta complejidad se convierte en un problema cuando la coordinación es ineficiente y la distancia institucional es grande.

La falta de un instrumento de planificación con vocación holística e integrador en el ámbito litoral similar a lo existente en la planificación hidrológica genera desajustes importantes y nuevos retos de cara a la integración del cambio climático.

Aunque todavía existe una gran distancia entre la presencia del cambio climático en la normativa actual y elaboración de medidas operativas y vinculantes, existen dos iniciativas catalanas en marcha que pretenden allanar el camino. Tanto la Llei de canvi climàtic, como la Llei d'ordenació litoral abren nuevas expectativas para mejorar en este campo, aunque si no se resuelve el encaje competencial se pueden reproducir las dinámicas presentes. En este compromiso ayudaría que hubiera un marco estratégico a nivel europeo que dotara de elementos estratégicos y metodológicos como hizo la Directiva Marco del Agua o está haciendo la Directiva Marco de Estrategia Marina pero para el ámbito estrictamente litoral.

\section{AGRADECIMIENTOS}

Proyecto PaiRisClima (CGL2014-55387-R) financiado por el Ministerio de Economia y Competitividad. Gobierno Español. 


\section{REFERENCIAS}

Allen, CR. Fontaine, JJ. Pope, KL. Garmestani, AS. (2011) Adaptive management for a turbulent future. Journal of Environmental Management, 92, 1339-1345.

Dietz, T. Ostrom, E. Stern, PC. (2003). The struggle to govern the commons. Science 302, 1907-1912.

Norton, B.G. (2005). Sustainability: a Philosophy of Adaptive Ecosystem Management. Chicago: University of Chigaco Press.

Ribot, E. (2008) El marc legal en matèria de riscos naturals a catalunya. En: Vilaplana, JM. Payàs, B. (Coords). Els Riscos Naturals a Catalunya. Informe del CADS 6. 1-144. Generalitat de Catalunya. 